\title{
Glial Fibrillary Acidic Protein (GFAP) Astrocytopathy Presenting as Mild Encephalopathy with Reversible Splenium Lesion
}

\author{
Charlotte Héraud · Nicolas Capet · Michaël Levraut · Robin Hattenberger · \\ Véronique Bourg $\cdot$ Pierre Thomas $\cdot$ Lydiane Mondot $\cdot$ Christine Lebrun-Frenay
}

Received: September 30, 2021 / Accepted: November 5, 2021 / Published online: November 29, 2021

(C) The Author(s) 2021

\begin{abstract}
Introduction: Autoimmune glial fibrillary acidic protein (GFAP) astrocytopathy is scarce and usually presents as meningoencephalomyelitis. Here, we offer the case of an atypical presentation of GFAP-astrocytopathy.
\end{abstract}

C. Héraud $(\bowtie) \cdot$ N. Capet · R. Hattenberger ·

V. Bourg · P. Thomas - C. Lebrun-Frenay

Service de Neurologie, Centre Hospitalier

Universitaire de Nice, 30 Voie Romaine, 06100 Nice,

France

e-mail: heraud.c@chu-nice.fr

N. Capet · M. Levraut · L. Mondot .

C. Lebrun-Frenay

Service de Neurologie, Centre Hospitalier

Universitaire de Nice, URC2A-URRIS, Université de

Nice Côte d'Azur, CRC SEP, 30 Voie Romaine, Nice,

France

M. Levraut

Service de Médecine Interne, Centre Hospitalier

Universitaire de Nice, 30 Voie Romaine, Nice,

France

L. Mondot

Service de Neuroradiologie, Centre Hospitalier

Universitaire de Nice, 30 Voie Romaine, Nice,

France
Case Presentation: We report the case of a 26-year-old woman admitted to our neurology department for a 3-week progressive and worsening neurologic picture, with secondary worsening. Initial imaging showed a Mild Encephalitis with Reversible Splenium of corpus callosum lesion (MERS). Full infectious and autoimmune workup then revealed positivity of GFAP antibodies, leading us to diagnose GFAP astrocytopathy.

Discussion: Our case is the first reported association between MERS and GFAP astrocytopathy in an adult patient. Clinical presentation of GFAP astrocytopathy usually includes various neurologic symptoms and can lead to misdiagnosis. 
Keywords: GFAP; Astrocytopathy; MERS; MRI

\section{Key Summary Points}

Our case is the first reported association between Mild Encephalitis with Reversible Splenium of corpus callosum lesion (MERS) and autoimmune glial fibrillary acidic protein (GFAP) astrocytopathy in an adult patient.

Our initial hypothesis was infectious meningoencephalomyelitis because of the rapid clinical worsening and lymphocytic meningitis associated with hyperproteinorachia.

GFAP antibody positivity in the cerebrospinal fluid (CSF) led us to diagnosis GFAP astrocytopathy, a rare inflammatory disorder of the CSF.

This case highlights the clinical, biologic, and radiologic diversity of the GFAP astrocytopathy presentation.

\section{INTRODUCTION}

Autoimmune glial fibrillary acidic protein (GFAP) astrocytopathy is a rare inflammatory disorder of the central nervous system, with diverse clinical presentations of acute onset. We report the case of GFAP astrocytopathy presenting with a radiologic picture of Mild Encephalitis with Reversible Splenium of corpus callosum lesion and lymphocytic meningitis. It is still difficult today to describe the pathophysiology of this autoimmune disorder. In $25 \%$ of cases, GFAP astrocytopathy is associated with neoplasia, which is to be thoroughly researched when diagnosing this affection. Diagnosis is currently based on clinical presentation, imaging (patients usually carry MRI abnormalities on T2-weighted sequences and post-gadolinium T1), and cerebrospinal fluid (CSF) analysis, as no reliable diagnostic criteria have been published.

\section{CASE PRESENTATION}

We report the case of a 26-year-old left-handed woman admitted to our neurology department for a 3-week progressive and worsening fatigue, fever, headaches, diplopia, and walking difficulties. Written informed consent was obtained from the patient before publication. She did not report any medical history except active

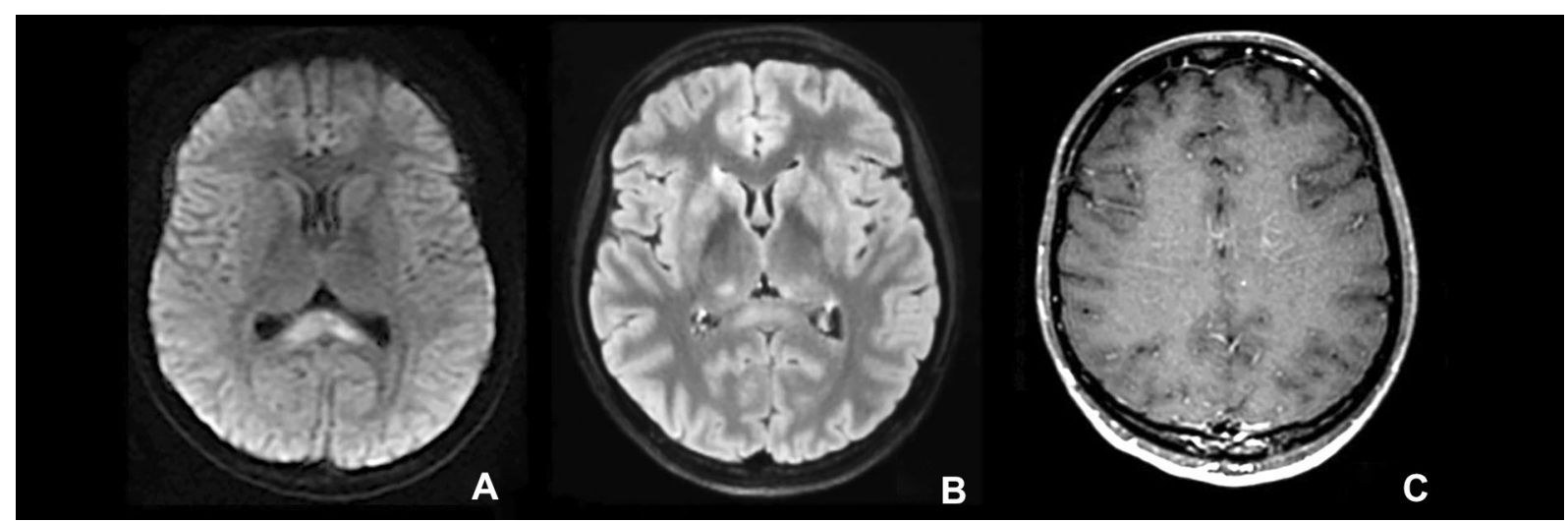

Fig. 1 A Axial DWI-weighted brain MRI showing bilateral and symmetrical corpus callosum hyperintensity defined as MERS. B Axial T2-FLAIR weighted brain MRI, bilateral and symmetrical corpus callosum hyperintensity.
C Axial post-gadolinium T1-weighted brain MRI, corpus callosum hypointensity, linear gadolinium enhancement of the Virchow-Robin perivascular spaces 


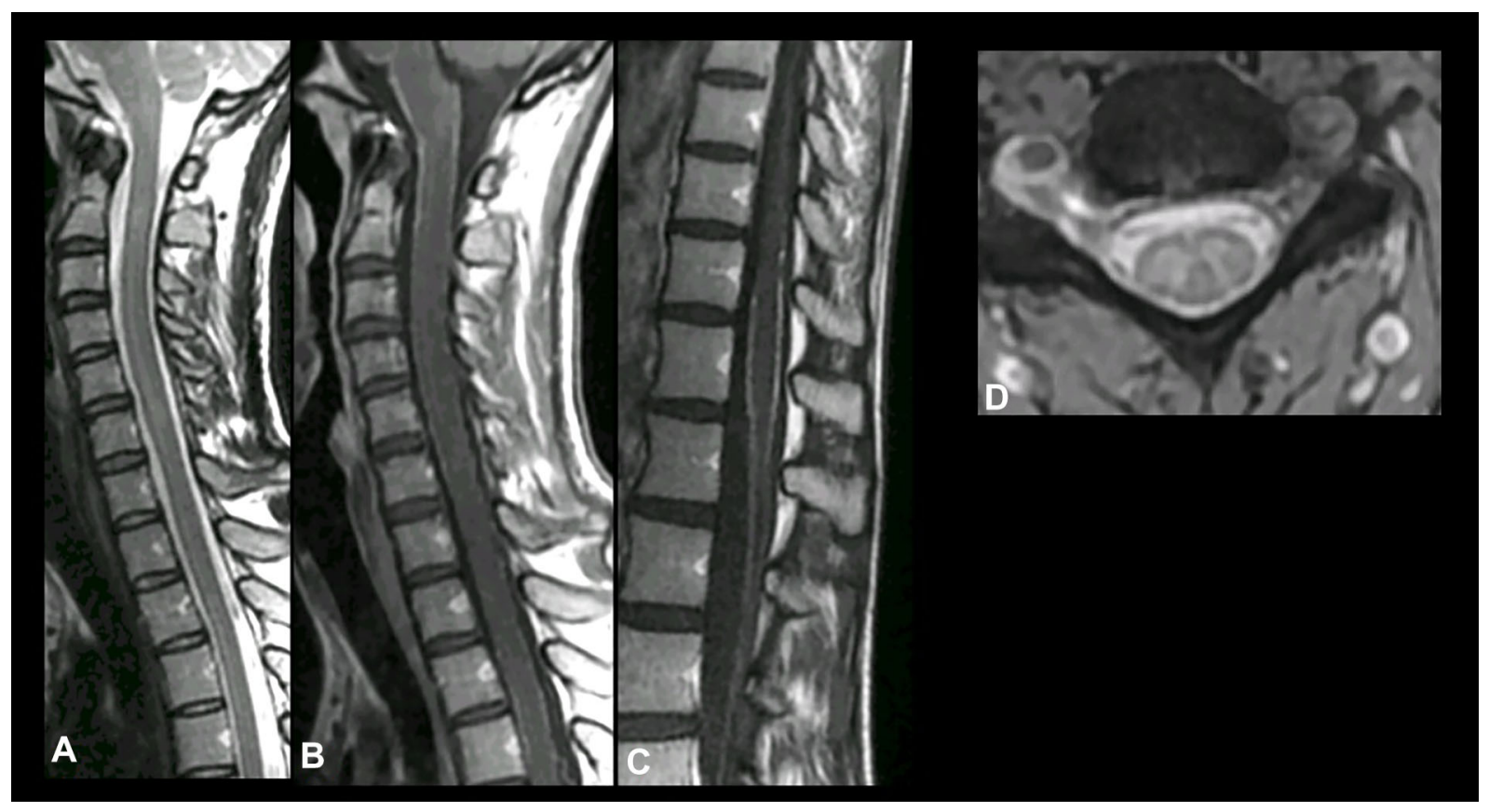

Fig. 2 A Sagittal T2-weighted cervico-dorsal MRI, hyperintensities on C2, C3, and C6 levels. B Sagittal T1weighted cervico-dorsal MRI after gadolinium injection, diffuse leptomeningeal gadolinium enhancement. C Sagittal post-gadolinium T1-weighted dorso-lumbar MRI, diffuse

smoking, not taking medications, and her vaccinations not being updated for 10 years. Physical examination revealed nausea, vomiting, and headaches without meningeal syndrome associated with multidirectional nystagmus, a callosum disconnection syndrome with right side apraxia and anosognosia, multiple cranial nerve palsy (left sixth cranial nerve, right facial and bilateral trigeminal hypoesthesia), and proprioceptive ataxia of the lower limbs. The brain MRI showed a diffusion-weighted imaging (DWI) and fluid-attenuated inversion recovery (FLAIR) hyperintensity of the corpus callosum suggestive of a Mild Encephalitis with Reversible Splenium of corpus callosum lesion (MERS), as shown in Fig. 1. The spinal cord MRI highlighted three lateral and posterior cervical hyperintensities on FLAIR weighted images suggestive of focal myelitis, and post-gadolinium MRI showed a diffuse leptomeningeal gadolinium enhancement (Fig. 2). CSF analysis showed a hyperproteinorachia and hypoglycorrhachia $\quad(1.51 \mathrm{~g} / \mathrm{l}$ and $1.93 \mathrm{mmol} / \mathrm{l}$, leptomeningeal gadolinium enhancement. D Axial T2weighted spinal cord MRI, right anterior spinal cord hyperintensity

$N<0.45 \mathrm{~g} / \mathrm{l}$ and $N \quad 2.20-4 \mathrm{~g} / \mathrm{l}$, respectively) associated with lymphocytic meningitis (121 white blood cells [WBC]/ $\mu 1,85 \%$ lymphocytes) with negative gram staining. Because of these first results, an infectious meningoencephalomyelitis was considered, and intravenous antiviral treatment (Aciclovir) was started with high-dose amoxicillin and cephalosporin. On day 4, while the patient was still under anti-infective agents, she experienced severe clinical worsening presented by altered consciousness, left facial paralysis, acute urinary retention, dysarthria, and complete visual loss in the left eye.

Another spinal tap was performed and showed an increase of CSF WBC count $(450 / \mu \mathrm{l}$, $77 \%$ lymphocytes) with persistently elevated CSF protein and low CSF glucose. Infectious workup including CSF polymerase chain reaction (PCR) for herpes simplex virus (HSV) 1 and 2 , enterovirus, varicella zoster virus (VZV), cytomegalovirus (CMV), tuberculosis, blood serology for human immunodeficiency virus 


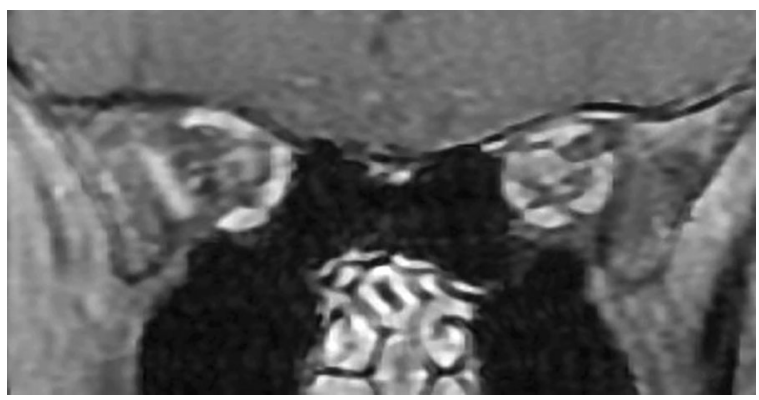

Fig. 3 Coronal post-gadolinium T1-weighted optic nerve MRI, leptomeningeal gadolinium enhancement of the optic nerves

(HIV), syphilis, Lyme, or intracellular bacteria, as well as CSF and blood cultures was negative. A second cerebral MRI highlighted the corpus callosum hyperintensity regression, confirming the MERS, but showed a leptomeningeal (especially in the cranial nerves and the medulla oblongata) gadolinium enhancement, particularly in the optic nerves (Fig. 3). Ophthalmologists concluded left optic neuritis. The patient then stopped anti-infective agents and started high-dose intravenous steroids at $1 \mathrm{~g} /$ per day for 5 days followed by a $2 \mathrm{mg} / \mathrm{kg} /$ day maintenance steroid therapy after new extensive blood and CSF immune analysis including anti-myelin oligodendrocyte (MOG) antibodies (Ab), antiaquaporin-4 Ab, and neuronal cell surface and intracellular $\mathrm{Ab}$ with anti-glial fibrillary acidic protein (GFAP) Ab. Blood analysis highlighted hyponatremia, $\mathrm{HBV}, \mathrm{CMV}, \mathrm{EBV}$, rubella, mumps, parvovirus, VZV, and measles immunization. We searched for associated autoimmune diseases because the lesion in the corpus callosum could not explain all of the symptoms the patient presented throughout her hospitalization. Immunologic workup including antinuclear antibodies showed no evidence of autoimmune disease. Moreover, the patient did not have any extra-neurologic symptoms that might have led us to suspect a systemic infection.

The full-body computed tomography (CT) scan and the 18-FDG positron emission tomography-CT were unremarkable. On day 29, a third MRI displayed near-total disappearance of the corpus callosum hyperintensity and leptomeningeal contrast enhancement persistence. On day 37 , while taking $1 \mathrm{mg} / \mathrm{kg} /$ day steroids, the patient was discharged from the hospital and was able to go home with outpatient rehabilitation while experiencing persistent diplopia, bilateral facial hypoesthesia, right facial paralysis, and gait disturbance, as well as needing intermittent bladder catheterization. Immunologic analysis of the CSF then showed positivity for anti-GFAP Ab. Because of the clinical presentation, clinical response to steroids, and CSF positivity for specific $\mathrm{Ab}$, an antiGFAP astrocytopathy diagnosis was retained. Therefore, steroid therapy was progressively tapered over 3 months. The patient was free from neurologic and cognitive symptoms at the 4-month follow-up visit, walked alone without difficulty, and did not report any sphincter disorder. As for imaging, we observed the almost disappearance of the corpus callosum hyperintensity and cranial nerve enhancement, as shown in Fig. 4. No new lesion was enhanced after gadolinium injection. Anti-GFAP antibodies tested positive for the second time in the CSF 3 months after hospitalization on March 2nd 2021.

\section{DISCUSSION}

Autoimmune glial fibrillary acidic protein (GFAP) astrocytopathy is an inflammatory disorder of the central nervous system (CNS). Diagnosis is performed with detection in CSF of IgG reactive with GFAP associated with meningoencephalomyelitis. GFAP autoimmune astrocytopathy can present at any age but is usually diagnosed in patients $>40$ years old, with a slight female predominance [1]. In 10\% of cases, children are affected. Glial fibrillary acidic protein is the main intermediate filament protein in astrocytes and is a significant cytoskeleton component. Pathophysiology of autoimmune GFAP astrocytopathy is unknown to this day. This astrocytopathy is an autoimmune disorder. However, 25\% of patients have GFAP astrocytopathy associated with a neoplasm (mainly ovarian teratomas) [2]. 


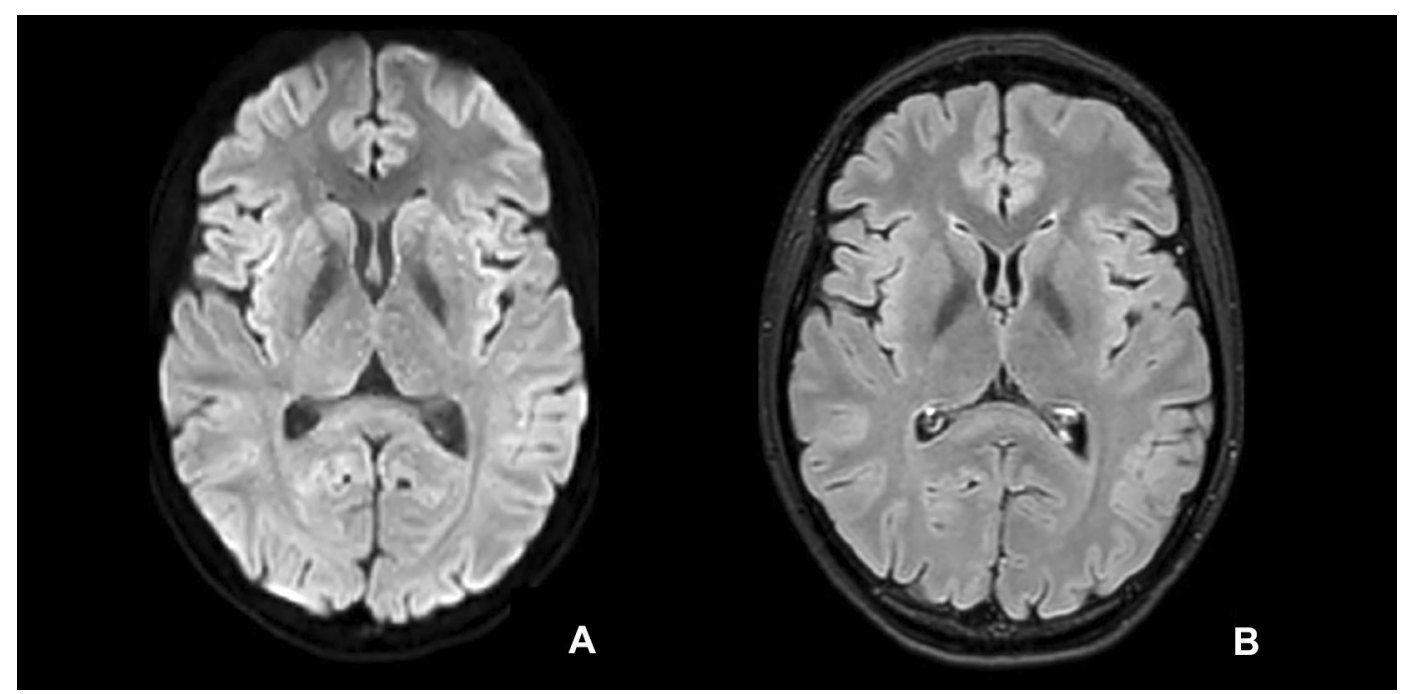

Fig. 4 A Axial DWI-weighted brain MRI at 4 months, the nearly total disappearance of the corpus callosum hyperintensity. B Axial T2-FLAIR weighted brain MRI at 4 months, the nearly total disappearance of the corpus callosum hyperintensity

Clinical presentation usually includes encephalopathy, seizures, psychiatric symptoms, area postrema syndrome [3], tremor, headache, myelitis, abnormal vision, and meningeal syndrome, along with rare movement disorders, neuropathy, or dysautonomia. In most cases, patients' symptoms appear with an acute or subacute onset [2]. Viral prodromes or flu-like symptoms are prevalent, preceding the neurologic symptoms. The disease may be monophasic or present relapsing courses, requiring prolonged treatment [2].

Patients usually carry MRI abnormalities, especially on T2-weighted sequences and postgadolinium T1. Lesions are typically observed in the subcortical white matter, basal ganglia, hypothalamus, brainstem, cerebellum, meninges, and ventricles. A characteristic lesion presents as a linear, radial perivascular enhancement pattern through the cerebral white matter because of the GFAP abundance in the peri-ventricular regions. Enhancement can also be observed in leptomeningeal and ependymal areas as well as in the form of punctate or serpentine lesions within the white matter. Myelitis is quite common in GFAP astrocytopathy, most frequently in the form of meningoencephalomyelitis. These spinal cord hyperintensities usually present as central longitudinally extensive lesions. Compared to aquaporin 4-associated (AQP4) myelitis, they show less edema and poorly defined margins. The GFAP astrocytopathy may be considered a differential diagnosis of neuromyelitis optica spectrum disorder (NMOSD) as it associates cerebral and medullar lesions and can affect the optical nerve [2].

Regarding biologic screening, most patients present lymphocyte-predominant elevation in white blood cells $\left(>50 \times 10^{6} / \mathrm{l}\right)$ and elevated protein levels $(>1 \mathrm{~g} / \mathrm{l})$ in CSF. Literature is relatively sparse on oligoclonal bands in CSF. However, we notice that one patient out of two has oligoclonal bands in CSF [1].

Our patient is relatively younger than the subjects in previous publications and represents the first reported association between MERS and GFAP astrocytopathy in an adult patient [4]. To date, only one case of MERS-GFAP association was described in a pediatric case [5]. Moreover, this case highlights the difficulties in the diagnostic workup while our patient presented with extensive myelitis and optic neuritis suggestive of NMOSD. However, flu-like symptoms preceding neurologic deficits, leptomeningeal MRI gadolinium enhancement, extensive myelitis, and lymphocytic meningitis are often seen in GFAP astrocytopathy. This case description 
highlights the complexity of GFAP astrocytopathy diagnosis and high risk of misdiagnosis. Our case description is the first MERS-GFAP described in an adult patient.

No reliable diagnostic criteria have been published for GFAP astrocytopathy to date. The risk of overdiagnosing is excellent if we rely on GFAP antibody positivity in the CSF due to false positives. The principal differential diagnosis for GFAP astrocytopathy is NMOSD, and complete remission after treatment with corticosteroids does not direct us towards NMOSD. As for myelin oligodendrocyte glycoprotein (MOG) antibody disorders, blood MOG Ab tested negative twice. Moreover, the presence in the CSF or serum of other antibodies is expected in the acute phase of the disease and may lead to misdiagnosis.

Based on clinical observations, treatment relies on corticosteroids for the first clinical manifestations. Intravenous immunoglobulins and plasma exchange may also be required [6]. Empirical data suggest a superiority of corticosteroids over intravenous immunoglobulins, which is why they are reserved for patients unresponsive to corticotherapy [7]. Long-term treatment depends on oral low-dose corticosteroids or immunosuppressants.

\section{CONCLUSION}

GFAP astrocytopathy is a tricky diagnosis as its clinical, biologic, and radiologic presentation can mimic various neurologic affections. GFAP antibody positivity in the CSF is not sufficient for astrocytopathy diagnosis. Relying on empirical observations, treatment is based on corticosteroids. Due to epidemiologic association with neoplasm (ovarian teratoma being the most frequent), exhaustive oncological workup is essential.

\section{ACKNOWLEDGEMENTS}

We thank the patient for her participation and her consent for publication.
Funding. No funding or sponsorship was received for this study or publication of this article.

Authorship. All named authors meet the International Committee of Medical Journal Editors (ICMJE) criteria for authorship for this article, take responsibility for the integrity of the work as a whole, and have given their approval for this version to be published.

Authors' Contributions. Charlotte Héraud and Michaël Levraut conceived and designed the draft of the manuscript. Nicolas Capet, Robin Hattenberger, Véronique Bourg, Pierre Thomas, and Christine Lebrun-Frenay critically revised the manuscript for important intellectual contents. Lydiane Mondot helped edit the imaging. Christine Lebrun-Frenay provided editorial assistance in the preparation of this article and gave the final approval of the version to be published. All authors have read and approved the manuscript.

Disclosures. Charlotte Héraud, Nicolas Capet, Michaël Levraut, Robin Hattenberger, Véronique Bourg, Pierre Thomas, Lydiane Mondot, and Christine Lebrun-Frenay do not have any conflict of interest.

Compliance with the Ethics Guidelines. Written informed consent was obtained from the patient before publication.

Data Availability. Data sharing does not apply to this article as no datasets were generated or analyzed during the current study.

Open Access. This article is licensed under a Creative Commons Attribution-NonCommercial 4.0 International License, which permits any non-commercial use, sharing, adaptation, distribution and reproduction in any medium or format, as long as you give appropriate credit to the original author(s) and the source, provide a link to the Creative Commons licence, and indicate if changes were made. The images or other third party material in this article are included in the article's Creative Commons licence, unless indicated otherwise in a credit 
line to the material. If material is not included in the article's Creative Commons licence and your intended use is not permitted by statutory regulation or exceeds the permitted use, you will need to obtain permission directly from the copyright holder. To view a copy of this licence, visit http://creativecommons.org/licenses/by$\mathrm{nc} / 4.0 /$.

\section{REFERENCES}

1. Kunchok A, Zekeridou A, McKeon A. Autoimmune glial fibrillary acidic protein astrocytopathy. Curr Opin Neurol. 2019;32(3):452-8.

2. Shan F, Long Y, Qiu W. Autoimmune glial fibrillary acidic protein astrocytopathy: a review of the literature. Front Immunol. 2018;5(9):2802.
3. Oger V, Bost C, Salah L, Yazbeck E, Maurey H, et al. Mild encephalitis/encephalopathy with reversible splenial lesion syndrome: an unusual presentation of anti-GFAP astrocytopathy. Eur J Paediatr Neurol. 2020;26:89-91.

4. Fang B, McKeon A, Hinson SR, Kryzer TJ, Pittock J, et al. Autoimmune glial fibrillary acidic protein astrocytopathy: a novel meningoencephalomyelitis. JAMA Neurol. 2016;73(11):1297-307.

5. Ciron J, Sourdrille F, Biotti D, Tchoumi T, Ruiz A, et al. Area postrema syndrome: another feature of anti-GFAP encephalomyelitis. Mult Scler J. 2020;26(2):253-5.

6. Issa N, Martin C, Dulau C, Camou F. Severe anti-GFAP meningo-encephalomyelitis following viral infection. Mult Scler Relat Disord. 2020;45:102448.

7. Heide E, Chaudhari A, Pirverdian A, Lai S, Courtney A. Failure of IVIG in steroid-responsive autoimmune glial fibrillary acidic protein astrocytopathy: a case report. Mult Scler Relat Disord. 2021;51:102933. 